\title{
STATISTICS OF HI EXTENTS OF IRREGULAR GALAXIES
}

\author{
G. LYLE HOFFMAN \\ Lafayette College \\ Easton, PA 18042-1782, USA
}

Using the 3.'2 Arecibo beam, we have mapped the HI envelopes of a sample of 70 irregular galaxies (Sdm, Sm, Im and BCD), including 45 from the Virgo Cluster Catalog, 14 Field galaxies at similar redshifts, and 11 Nearby dwarfs chosen to give a complete sample out to $6 \mathrm{Mpc}$, within the Arecibo declination range. To explore correlations among variables characterizing the size and dynamics of these galaxies, we have combined this sample with all other mappings (multiple single beams and synthesis array) of irregulars and of spiral galaxies spanning the same redshift range (out to about $20 \mathrm{Mpc}$ ) available in the literature. In all, there are 128 mapped dwarfs and 119 mapped spirals in this dataset. We obtain the following correlations: $r_{H, \max }=(2.75 \pm .15) r_{25}^{(0.890 \pm .034)}$ where both HI and optical radii are in kpc; $L_{B}=(3.59 \pm .80) \times 10^{6} r_{g m}^{(2.70 \pm .11)}$ where $L_{B}$ is in solar luminosities and $r_{g m}$ is the geometric mean of $r_{H, \max }$ and $r_{25} ; L_{B}=(125 \pm 36) V_{c}^{(3.62 \pm .19)}$ where the rotation speed is in $\mathrm{km} \mathrm{s}^{-1} ; M_{H}=(9.3 \pm 1.1) \times 10^{6} r_{g m}^{(1.977 \pm .056)}$ where the HI mass is in solar units; and $L_{B}=\left(1.9_{-1.1}^{+2.7}\right) \times 10^{-3} M_{d y n}^{(1.164 \pm .043)}$ where $M_{d y n} \equiv V_{c}^{2} r_{g m} / \mathrm{G}$.

Arguably the most important result of our survey is the uniqueness of the Giovanelli and Haynes "protogalaxy" HI 1225+01 in any plot of HI radius vs. optical radius or luminosity. DDO 154 and DDO 137 are seen in such plots to be on the tail of a continuous distribution while HI $1225+01$ sits alone. Objects with HI radius more than 10 times the optical extent $\left(r_{25}\right)$ are evidently quite rare. The scatter for dwarf irregulars is larger than that for spirals in all cases, apparently in part intrinsically so, and increases as the systems become smaller. Nevertheless, it is apparent that the relationship for dwarfs fits smoothly onto the extension of that for spirals with no pronounced dislocation or change in slope. Further implications will be discussed in a forthcoming paper. 